\title{
Revealing microbial recognition by specific antibodies
}

\author{
Áurea Simón-Soro', Giuseppe D’Auria', M. Carmen Collado², Mária Džunková', Shauna Culshaw ${ }^{3}$ and Alex Mira ${ }^{\text {** }}$
}

\begin{abstract}
Background: Recognition of microorganisms by antibodies is a vital component of the human immune response. However, there is currently very limited understanding of immune recognition of $50 \%$ of the human microbiome which is made up of as yet un-culturable bacteria. We have combined the use of flow cytometry and pyrosequencing to describe the microbial composition of human samples, and its interaction with the immune system.

Results: We show the power of the technique in human faecal, saliva, oral biofilm and breast milk samples, labeled with fluorescent anti-IgG or anti-IgA antibodies. Using Fluorescence-Activated Cell Sorting (FACS), bacterial cells were separated depending on whether they are coated with $\lg A$ or $\lg G$ antibodies. Each bacterial population was PCR-amplified and pyrosequenced, characterizing the microorganisms which evade the immune system and those which were recognized by each immunoglobulin.
\end{abstract}

Conclusions: The application of the technique to healthy and diseased individuals may unravel the contribution of the immune response to microbial infections and polymicrobial diseases.

Keywords: Immunoglobulin, Flow cytometry, Pyrosequencing, 165 rRNA, Opsonization, Human microbiome

\section{Background}

There are extensive data describing the human microbiome, and germ free animal models demonstrate its intricate relationship with the host [1]. However, less is understood about this relationship in humans, either in health or disease. The coating of microorganisms by antibodies may promote defense against infection, and regulate the immune response to the microbiota to limit potentially damaging responses, thus maintaining homeostasis in human-associated microbial communities $[1,2]$. The recognition of microbes by different immunoglobulins (Igs) plays a vital role in the host-microbiome relationship [3], but the associations between Ig classes and specific groups of bacteria and fungi remain relatively poorly characterized [4]. Flow cytometry allows the separation of bacterial cells according to their population structure $[5,6]$ and to the fluorescence emitted by antibodies specifically bound to different human Igs $[7,8]$.

\footnotetext{
* Correspondence: mira_ale@gva.es

'Department of Health and Genomics, FISABIO Foundation, Center for Advanced Research in Public Health, Avda. Cataluña 21, 46020 Valencia, Spain

Full list of author information is available at the end of the article
}

These labeled populations of bacteria can be characterized by second-generation sequencing of PCR-amplified microbial rDNA genes, to provide a description of the bacterial and fungal diversity and taxonomic composition [9].

We have applied a combination of flow cytometry cell sorting coupled with pyrosequencing to identify the bacteria that are coated with specific antibodies, avoiding the sample bias imposed by bacterial culture (Fig. 1). We show the potential of this mixed approach by estimating the proportion of Ig-coated bacteria in saliva, faecal, oral biofilm and breast milk samples, as well as identifying the specific bacterial genera that are either coated and uncoated by different Igs.

\section{Methods}

\section{Sample collection and processing}

All donors signed informed consent and the sampling protocol was approved by the Dirección General de Salud Pública (DGSP) ethical committee (Valencian Health Authority, Spain) for the faecal and oral samples; and the Bioethics Subcommittee of Consejo Superior de Investigaciones Científicas (CSIC) for the breast milk samples. For the oral samples, unstimulated saliva samples and supragingival dental plaque from 5 individuals 


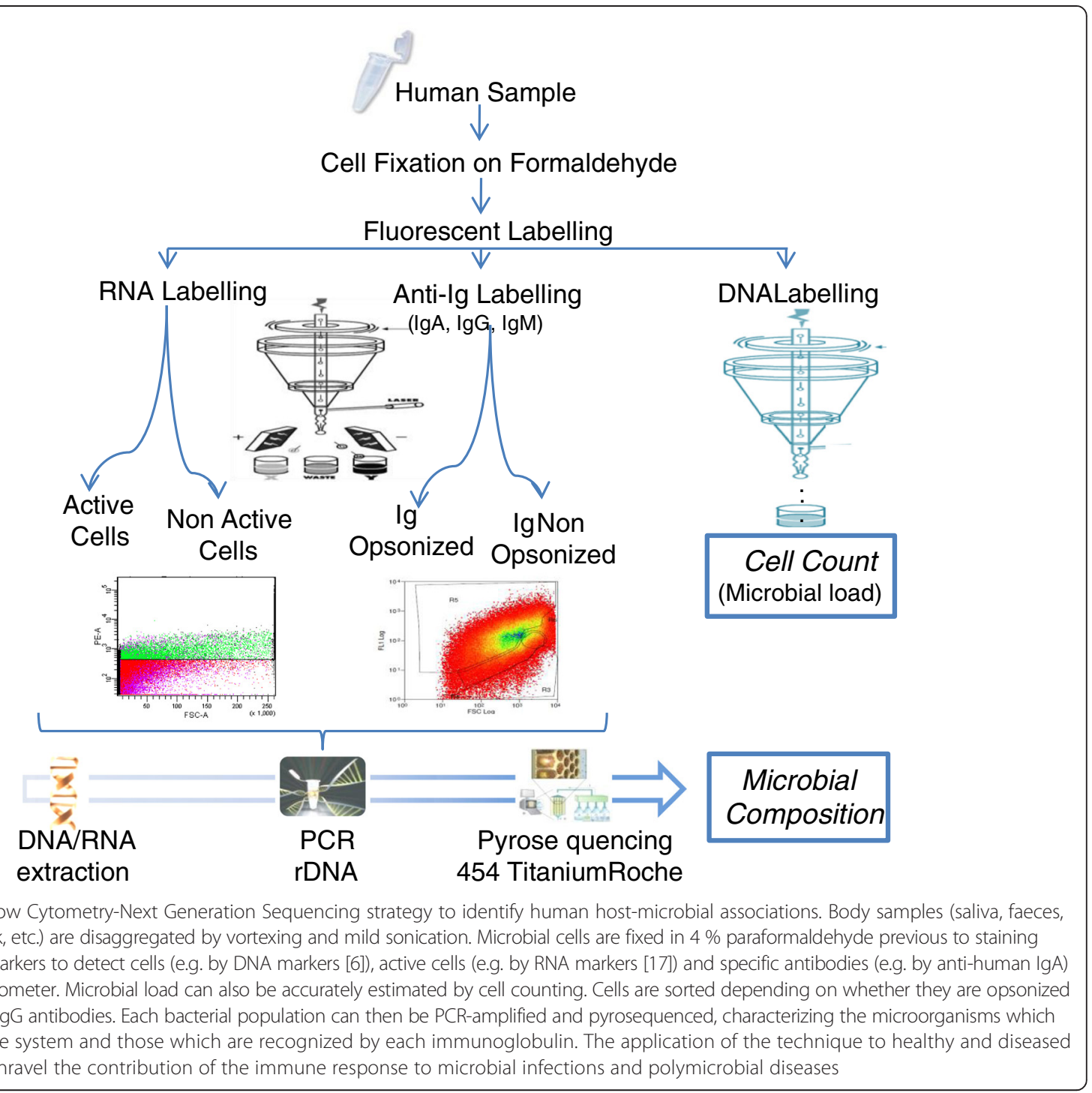

that had never suffered from dental caries and 4 individuals with active caries were collected in sterile tubes $24 \mathrm{~h}$ after tooth brushing. Six faecal samples were stored in RNALater within $2 \mathrm{~h}$ from sampling and stored at -20 until fixation in formaldehyde. Healthy volunteer mothers with term deliveries were given written instructions for standardised collection of breast milk samples. Before sample collection, the breast was cleaned with an iodine swab to reduce bacteria residing on the skin, and breast milk was collected manually and after discarding first drops collected into a sterile milk collection unit, then immediately frozen and stored at $-20{ }^{\circ} \mathrm{C}$ until analysis. A total of 12 breast milk samples corresponding to colostrum and mature milk were collected. All samples were centrifuged at $7500 \mathrm{~g}$ for $7 \mathrm{~min}$ to collect microbial cells and washed twice in physiological solution $(\mathrm{NaCl} 0.9 \%)$. Cells were immediately fixed in $4 \%$ paraformaldehyde overnight at $4{ }^{\circ} \mathrm{C}$. Fixed cells were washed twice and stored at $-20{ }^{\circ} \mathrm{C}$ in $50 \%$ ethanol until use. On the experiment day, samples were washed in sterile saline solution and disaggregated $20 \mathrm{~s}$ in a sonicator bath, model Raypa VCI-50 at low ultrasound intensity.

\section{Flow cytometry}

Samples were suspended in sterile saline solution with $5 \%$ albumin to prevent non-specific antibody binding, then stained with (i) anti-human IgA or IgG labelled with FITC (Invitrogen catalog \# A18782 and A18806); and (ii) the DNA-binding fluorophor SYTO62 (Invitrogen catalog \# S11344) according to the manufacturer instructions. Anti-mouse IgA or IgG labeled with FITC (Invitrogen catalog \# M31101 and A24525) were used for isotype controls.

Cell sorting was performed with the MoFloTM XDP flow cytometer (Beckman Coulter Inc.) using Argon $488 \mathrm{~nm}$ (blue) laser (200 $\mathrm{mW}$ power) and the $635 \mathrm{~nm}$ 
(red) diode laser (25 $\mathrm{mW}$ power) as light sources. The lasers were aligned using Flow-CheckTM $(10 \mu \mathrm{m})$ and Flow-SetTM $(3 \mu \mathrm{m})$ fluorospheres (Beckman Coulter, Inc.). Emission filters were 520/30 for FITC and 680/30 for SYTO62 respectively. Proper fluorescent labeling was assessed by fluorescence and confocal microscopy (Additional file 1: Figure S1). Cells were separated according to their fluorescence in both the FITC and SYTO62 channels (Ig-coated bacteria) or the SYTO62 channel only (non-coated bacteria).

\section{Taxonomic identification}

DNA from the Ig-coated and non-coated fractions with more than 5000 cells was extracted using the MasterPure $^{\mathrm{Tu}}$ Complete DNA and RNA Purification Kit (Epicentre Biotechnologies), following the manufacturer's instructions, with the addition of a lysozyme treatment [10]. The 16S rRNA gene was amplified using universal bacterial primers $8 \mathrm{~F}$ and 533R with sample-specific barcodes, as previously described [10]. Purified PCR products were mixed in equimolar amounts and sequenced using the 454 GS-FLX pyrosequencer (Titanium chemistry, Roche). The resulting $16 \mathrm{~S}$ rRNA reads were end-trimmed in 10 bp sliding windows with average quality value $>20$, then length $(200 \mathrm{pp})$ and quality filtered (average $\mathrm{Q}>20$ ). Taxonomic assignments were performed with the RDP classifier [11], and to estimate total diversity, sequences were clustered at $97 \%$ nucleotide identity over $90 \%$ sequence alignment length to obtain rarefaction curves. Principal Coordinates Analysis (PCoA) was performed with FastUnifrac [12], comparing the 16S-estimated composition with a phylogenetic approach that takes into account both taxonomically assigned and unassigned reads.

\section{Results and discussion}

Formaldehyde-fixed samples were stained with (i) antihuman IgA or IgG labelled with FITC; and (ii) the DNA-binding fluorophor SYTO62. Proper fluorescent marking was confirmed by fluorescence and confocal laser scanning microscopy (Additional file 1: Figure S1). Anti-mouse IgA or IgG, of identical isotype to the antihuman antibodies, labeled with FITC, (Invitrogen) were used to control for non-specific binding. Cells were separated in the flow cytometer according to their fluorescence in both the FL1-FITC and FL8-SYTO62 channels (Ig-coated bacteria) or the SYTO62 channel only (noncoated bacteria). DNA from the Ig-coated and noncoated fractions was extracted and the $16 \mathrm{~S}$ rDNA gene amplified and pyrosequenced, in order to describe bacterial composition of the sorted populations. An outline of the experimental pipeline is described in Fig. 1. The density and number of cells that could be sorted from each fraction varied among samples (Additional file 2: Table S1). Although PCR and subsequent pyrosequencing was achieved with as few as 5000 sorted bacteria, a larger number of cells is recommended for accurate description of microbial composition [13].

When a frequency histogram was built depending on the anti-Ig marker fluorescence, a bi-modal distribution was typically observed, with a small peak at low fluorescence and a larger peak at high fluorescence intensities, for both IgA and IgG (Fig. 2). This suggests that only a low proportion of bacteria are apparently ignored by, or

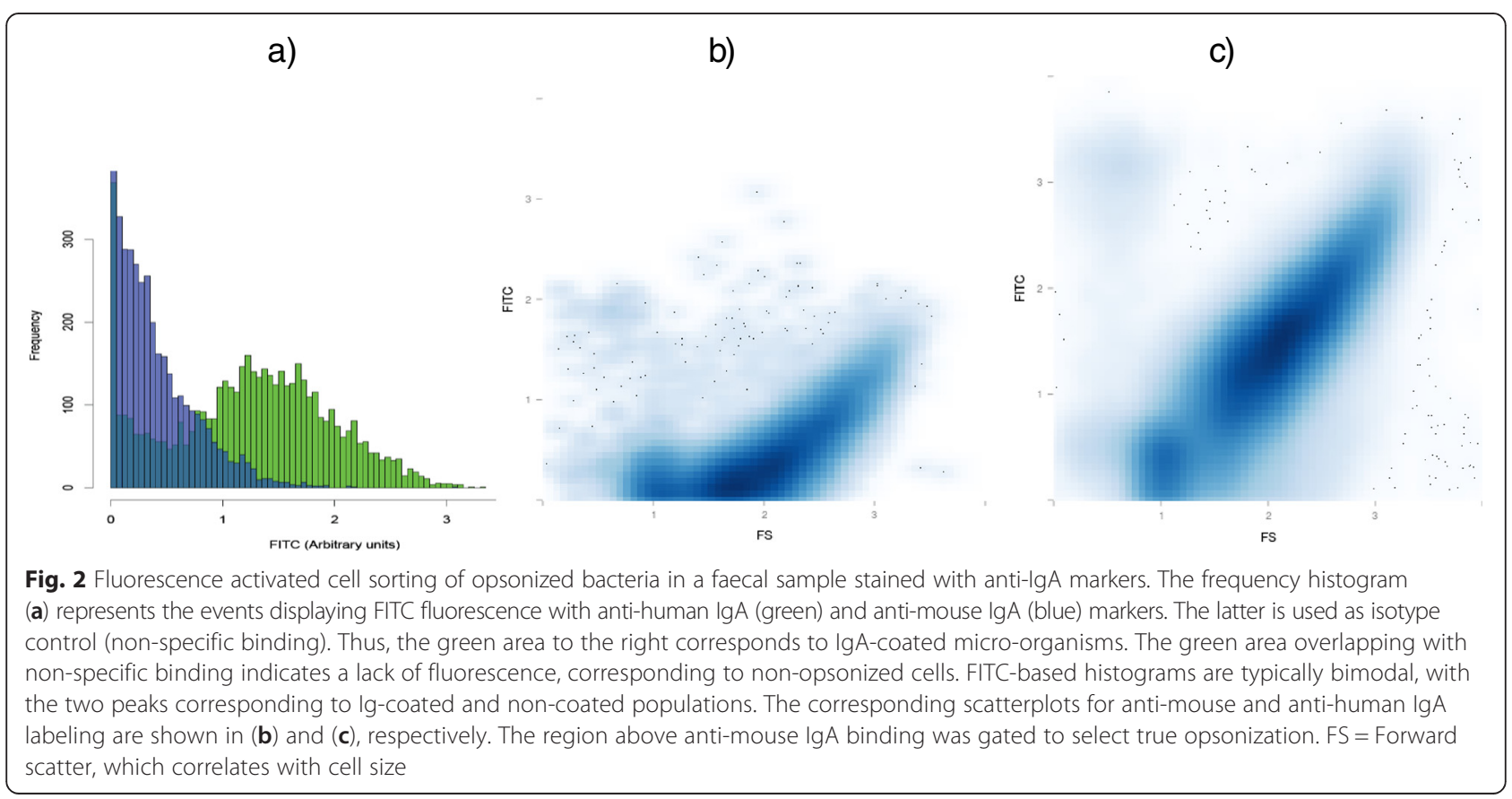


able to evade the humoral immune system. Specifically, the average proportion of IgA-opsonized bacteria in the subjects analyzed in the current proof of concept study ranged from $52.3 \%$ for faecal samples, $73.6 \%$ for saliva, $78.4 \%$ for the oral biofilm, and $63.7 \%$ for breast milk (Fig. 3, Additional file 2: Table S1). An even larger proportion of bacteria were coated with IgG; $93.8 \%$ for saliva, and $92.1 \%$ for the oral biofilm. This could potentially reflect a biological difference in the IgG/IgA antibodies specificity in oral samples, but different efficiencies in anti-human Ig antibodies binding or in fluorescence emission cannot be ruled out. In previous studies, $45 \%$ of faecal bacteria were found to be opsonized by IgA in samples from healthy donors, a proportion that increased to $69 \%$ under inflammatory episodes [14]. However, given that the number of secreted IgA was estimated to be $10^{7}$ times higher than the number of gut microbes [15], higher proportions can potentially be achieved. The greater proportion of opsonized bacteria detected in the oral samples analyzed in the current study is intriguing and could reflect local immune regulation requirements or an antibody mediated defense to limit microorganisms binding the gastrointestinal tract. It is also interesting to note a significantly higher Igcoating level in saliva and oral biofilm samples from healthy individuals compared to caries-bearing patients (Fig. 3). This indicates that the immune activity in caries-free individuals could be more competent than in patients with compromised oral health.

Pyrosequencing of Ig-marked and unmarked cells identified which bacteria were opsonized and non-opsonized by specific antibodies. In both saliva, faeces, oral biofilm and breast milk samples, the frequency of many bacterial genera in the two sorted fractions was different, suggesting regional differences and a particular affinity of the antibody for some microorganisms (Fig. 4). As a consequence, specific bacteria appear to be able to evade opsonization, including Escherichia and Stenotrophomonas in the gut, or Enterococcus and Prevotella in breast milk. Others, like Veillonella and Fusobacterium, appear always opsonized
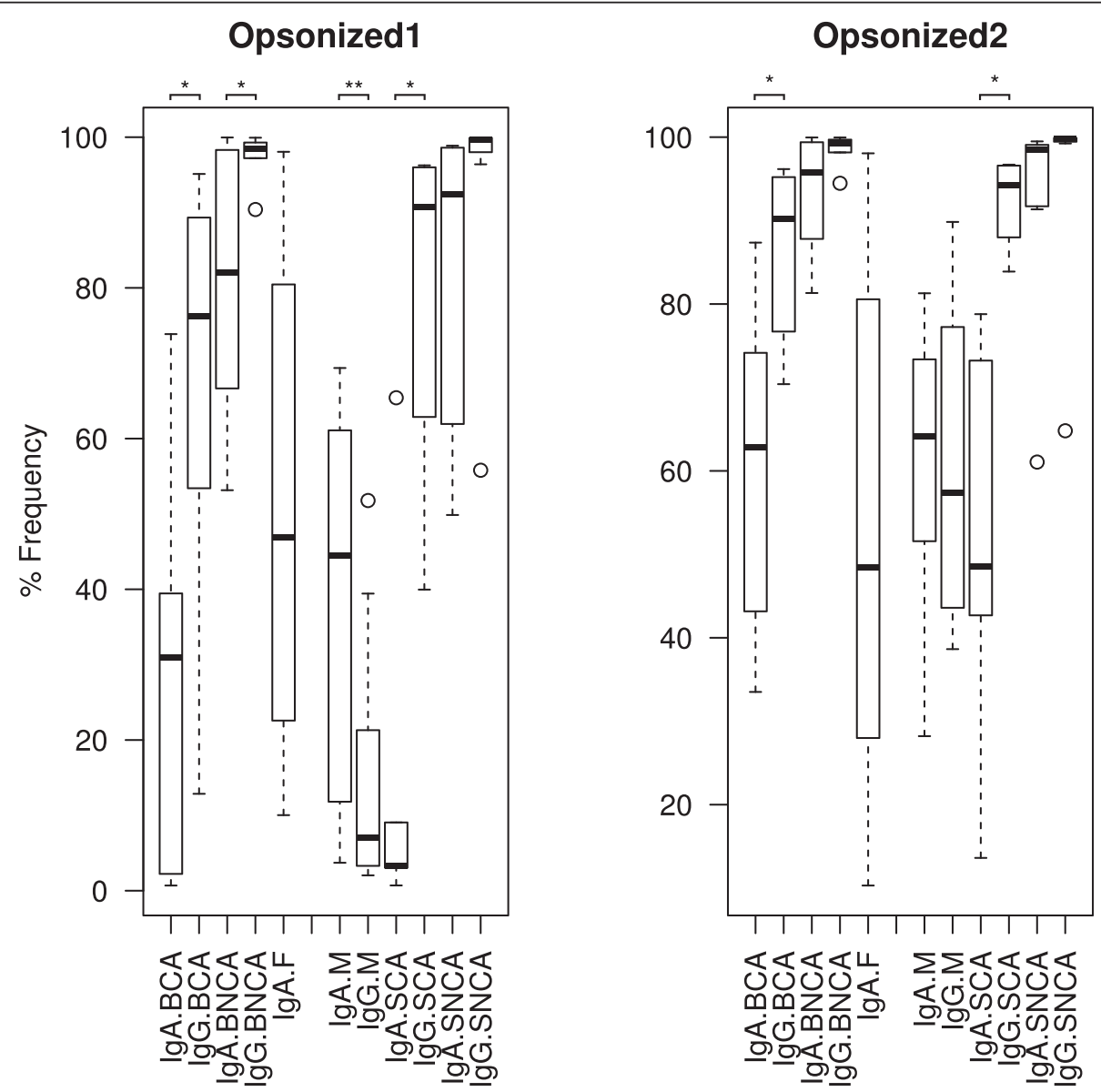

Fig. 3 lg-coating levels in different human samples. Boxplots show the mean values and variation in IgA- and IgG-opsonization levels for oral biofilm (B), milk (M), fecal samples (F), and saliva (S). Asterisks indicate statistically significant differences between IgA and IgG coating (Wilcoxon test, $p<0.05)$. For saliva and oral biofilm, samples from Caries-free (NCA) and Caries-bearing (CA) individuals are available. Data are shown for a conservative (Opsonized 1) and a non-conservative (Opsonized 2), upper estimate of Ig-coating. Individual data are shown in Additional file 2: Table S1 


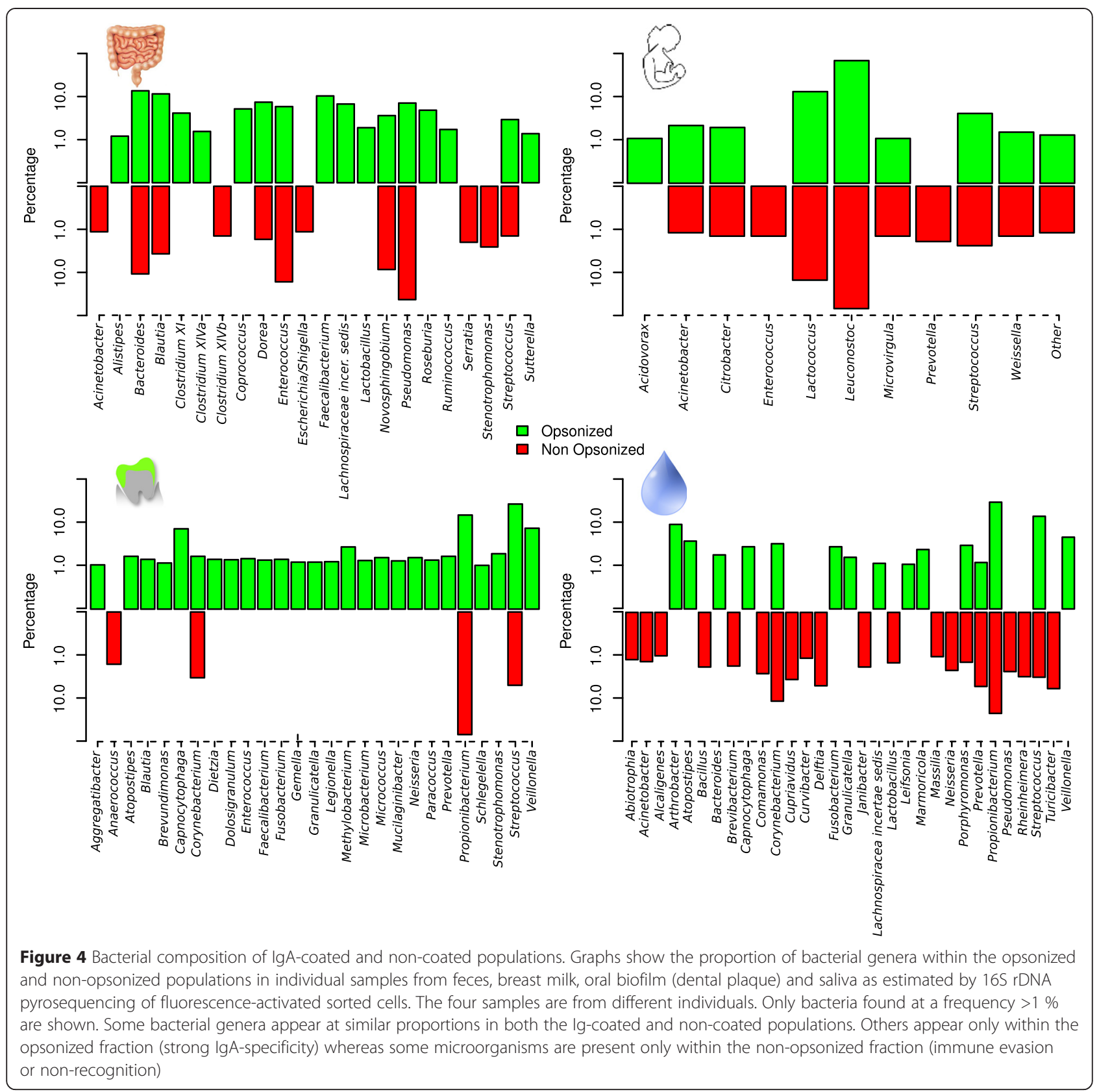

both in saliva and dental plaque. In the oral biofilm samples, most bacterial genera appeared to be IgA-coated.

The sum of the proportion of IgA- and IgG coated bacteria for the same samples was in most cases higher than $100 \%$, indicating that a large proportion of cells were coated by both immunoglobulins, as suggested by other studies $[16,17]$. However, the bacterial composition in the IgA- and IgG-coated fractions was different (Fig. 5). As shown by a Principal Coordinates Analysis in saliva samples, the composition of the IgA- and IgGopsonized fractions did not cluster together. In addition, the opsonized and non-opsonized populations occupied different positions in the PCoA space, indicating that the taxonomic groups coated by antibodies are different from those that are ignored or undetected by them. For instance, the genera Delftia or Propionibacterium were found at low frequencies in the saliva samples, but were highly opsonized by both IgA and IgG (Fig. 5a). Streptococcus, on the other hand, was very frequent in the same saliva samples, but moderately opsonized. Differential opsinization was found for Haemophilus, which was found to be coated by IgG but not IgA (Fig. 5a). Data for saliva samples from individual CA021 show that the most common genera in the IgA-opsonized fraction were Propionibacterium, Streptococcus, Arthrobacter, Veillonella and Atopostipes, whereas the latter three were absent in the non-opsonized 


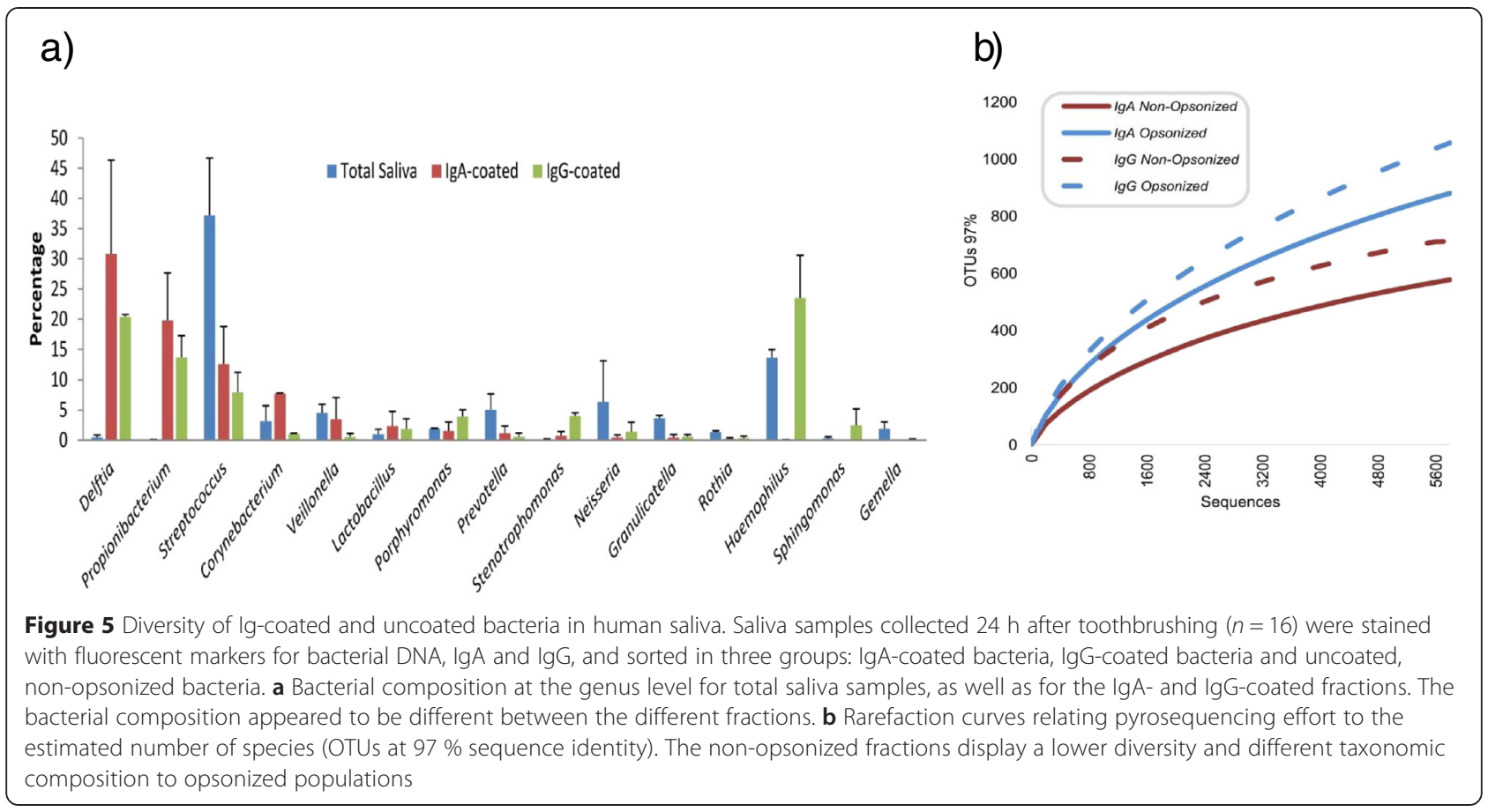

fraction (Fig. 4). In the future, the sequencing of IgA-, IgGand IgM-coated microbes in larger numbers of samples should confirm whether there is Ig-specific opsonization.

Finally, rarefaction curves of species-level bacterial diversity show that the opsonized population is more diverse than the non-opsonized one (Fig. 5b). In future studies, we anticipate that the sequencing of the nonopsonized fractions will reveal those micro-organisms that are undetected or ignored by specific antibodies. Although the current work was done with titanium chemistry FLX pyrosequencing and sequences were under $500 \mathrm{bp}$ long on average, current advances in this and other technologies are expected to allow read lengths over 900 bp shortly, allowing taxonomic assignment at the species level. This will no doubt be necessary for accurate description of antibody-microbial specificity, as current read lengths are mainly reliable at the genus level [18].

Another aspect that can readily be observed in flow cytometry scatter plots in environmental samples is the presence of aggregated populations as evidence by their larger size and specific shapes [5]. Our own observations in human samples through fluorescence and confocal microscopy revealed that some of those large-size clusters are bacterial aggregates and others are formed by bacteria bound to host cells like detached buccal epithelial cells. These aggregates can by sorted and subsequently identified by $16 \mathrm{~S}$ rDNA pyrosequencing (Additional file 3: Figure S2). In individual CA060, for instance, $70 \%$ of a bacterial aggregate in a saliva sample was found to be formed by Porphyromonas, Streptococcus, Prevotella, Propionibacterium, Veillonella, and unidentified Bacteroidetes. This approach paves the way to unravel the nature of bacterial aggregation in body fluids with important repercussion for active and passive immunization approaches and novel antimicrobial strategies. For instance, aggregated microorganisms may be less accessible to antibodies and partially escape opsonization.

The mixed FACS-pyrosequencing approach presented here can also be applied to identify fungi, by using fungalspecific fluorescent markers and subsequent sequencing of PCR-amplified fungal ITS or 28S rRNA regions [19]. In addition, an RNA-binding fluorophor like pyronin can be used to quantify, separate and sequence-identify active bacteria $[6,17]$. In our saliva samples $(n=6)$, 31-43\% of bacteria appeared to be marked by pyronin, suggesting that a large portion of organisms in the oral cavity can be transient or inactive (Additional file 1: Figure S1). In the future, marking of IgA and IgG with different fluorescent markers could be used in the same sample, in order to distinguish individual cells coated by both of these antibodies. Finally, micro-organisms cell counts can be used to accurately calculate bacterial and fungal load, which can be related to the body fluid chemical and biological components. That way, features of the immune response can be associated to microbial composition and density, providing insights about functioning of the immune system and suggesting potential biomarkers of health and disease conditions. 


\section{Conclusions}

The approach presented here involves the identification of Ig-detected and ignored microbes in healthy and diseased individuals $[14,20]$. This approach offers novel insights into understanding host-microbe homeostasis in health and its disruption in myriad diseases, ranging from oral diseases (e.g. dental caries or periodontal disease) to gut disorders (e.g. Crohn's disease, ulcerative colitis, or irritable bowel disease [20]) and even Ig-recognition of tumor cells. Considering immune recognition and opsonization in healthy individuals as a reference, deviations from that balanced microbe-immune interaction can potentially be related to microbial-mediated disorders, and the characterization of individual-specific opsonization profiles may also prove fruitful in diagnostic and therapeutic strategies for personalized medicine.

\section{Additional files}

Additional file 1: Figure S1. Staining of microbial cells for Fluorescence Activated Cell Sorting. Confocal laser microscopy images of saliva samples collected $1 \mathrm{~h}$ after toothbrushing from individual CATV01 stained with different fluorescent markers. (a) Saliva sample stained with the fluorophors SYTO62 (DNA labeling, red fluorescence) and FITC (anti-human IgM, green fluorescence). Image corresponds to the transversal section Z10, using a $63 \times$ magnification and a 1.5 zoom. (b) Saliva stained with the RNA-binding fluorophor Pyronin-Y (RNA labelling, red fluorescence). The intensity of fluorescence can be related to RNA production and therefore to cell activity and both active and non-active cells are observed. The image is a projection of all transversal sections in the sample, performed at $63 x$ magnification with a 3.0 zoom in a Leica HCX PLAPO confocal microscope. In both cases, stained and unstained bacteria can be separated by fluorescence detection cell sorting in order to describe the corresponding microbial populations by rRNA PCR and pyrosequencing

Additional file 2: Table S1. Estimated number of Ig-coated cells as detected by Fluorescence-Activated Cell Sorting in saliva, oral biofilm, faeces and breast milk human samples.

Additional file 3: Figure S2. Identification of bacterial composition in aggregates. The scatterplot shows microbial cells in a saliva sample according to their size ( $X$ axis) and the IgG coating, as indicated by their FITC fluorescence using anti-human IgG markers ( $Y$ axis). The large-size aggregate (indicated with a black circle) was separated by fluorescenceactivated cell-sorting and its DNA pyrosequenced after PCR of the $16 \mathrm{~S}$ rRNA gene, describing its bacterial diversity at the genus taxonomic level. The bacterial populations displaying FITC fluorescence values below 10 correspond to non-opsonized cells.

\section{Competing interests}

The authors declare that they have no competing interests.

\section{Authors' contributions}

AM, AS, GD and MCC conceived the study and participated in its design. AS and MD carried out the molecular and sequencing work, and AM, AS, MD and MCC performed the flow cytometry experiments. SC contributed to improve the flow cytometry protocols and helped to analyze and interpret the data. AM coordinated and wrote the manuscript. All authors read, corrected and approved the final manuscript.

\section{Acknowledgments}

This work was supported by projects BIO2012-40007 and Consolider CSD2009-0002 and CSD2007-00063 from the Spanish Ministry of Economy and Competitiveness; by grant CP09/00049 Miguel Servet, Instituto de Salud Carlos III, Spain; and by projects 071/2011 AP-034/11 from Generalitat
Valenciana. Flow Cytometry cell sorting was carried out using facilities at the Servei Central de Suport a la Investigació Experimental (SCSIE), University of Valencia.

\section{Author details}

${ }^{1}$ Department of Health and Genomics, FISABIO Foundation, Center for Advanced Research in Public Health, Avda. Cataluña 21, 46020 Valencia, Spain. ${ }^{2}$ The Institute of Agrochemistry and Food Technology, Spanish National Research Council (IATA-CSIC), 46980 Valencia, Spain. ${ }^{3}$ Infection and Immunity Research Group, Glasgow Dental School, School of Medicine, College of Medical, Veterinary and Life Sciences, University of Glasgow, Scotland, UK.

Received: 27 May 2014 Accepted: 29 May 2015

Published online: 02 July 2015

\section{References}

1. Hooper LV, Littman DR, Macpherson AJ. Interactions between the microbiota and the immune system. Science. 2012;336:1268-73.

2. Mshvildadze M, Neu J. The infant intestinal microbiome: friend or foe? Early Hum Dev. 2010;86 Suppl 1:67-71.

3. Brandtzaeg P. Homeostatic impact of indigenous microbiota and secretory immunity. Benef Microbes. 2010;1:211-27.

4. Woof JM, Mesteck J. Mucosal immunoglobulins. Immunol Rev. 2005;206:64-82.

5. Koch C, Günther S, Desta AF, Hübschmann T, Müller S. Cytometric fingerprinting for analyzing microbial intracommunity structure variation and identifying subcommunity function. Nat Protoc. 2013;8:190-202.

6. Peris-Bondia F, Latorre A, Artacho A, Moya A, D'Auria G. The active human gut microbiota differs from the total microbiota. PloS One. 2011;6, e22448.

7. Tsuruta T, Inoue R, Iwanaga T, Hara H, Yajima T. Development of a method for the identification of S-IgA-coated bacterial composition in mouse and human feces. Biosci Biotechnol Biochem. 2010;74:968-73.

8. Tsuruta T, Inoue R, Tsukahara T, Nakamoto M, Hara H, Ushida K, et al. Commensal bacteria coated by secretory immunoglobulin $\mathrm{A}$ and immunoglobulin $\mathrm{G}$ in the gastrointestinal tract of pigs and calves. Anim Sci J. 2012;83:799-804

9. Human Microbiome Project Consortium. Structure, function and diversity of the healthy human microbiome. Nature. 2012;486:207-14.

10. Simón-Soro A, Tomás I, Cabrera-Rubio R, Catalan MD, Nyvad B, Mira A. Microbial geography of the oral cavity. J Dent Research. 2013;92:616-21.

11. Wang Q, Garrity GM, Tiedje JM, Cole JR. Naive Bayesian classifier for rapid assignment of rRNA sequences into the new bacterial taxonomy. Appl Env Microbiol. 2007;73:5261-7.

12. Lozupone C, Hamady M, Knight R. UniFrac-an online tool for comparing microbial community diversity in a phylogenetic context. BMC Bioinformatics. 2006;7:371.

13. Biesbroek G, Sanders EA, Roeselers G, Wang X, Caspers MP, Trzciński K, et al. Deep sequencing analyses of low density microbial communities: working at the boundary of accurate microbiota detection. PLoS One. 2012;7, e32942.

14. van der Waaij LA, Kroese FG, Visser A, Nelis GF, Westerveld BD, Jansen PL, et al. Immunoglobulin coating of faecal bacteria in inflammatory bowel disease. Eur J Gastroenterol Hepatol. 2004;16:669-74.

15. Conley ME, Delacroix DL. Intravascular and mucosal immunoglobulin A: two separate but related systems of immune defense? Ann Intern Med. 1987;106:892-9.

16. Green GA. Autologous IgM, IgA, and complement binding to sickle erythrocytes in vivo. Evidence for the existence of dense sickle cell subsets. Blood. 1993;82:985-92.

17. D'Auria G, Peris-Bondia F, Džunková M, Mira A, Collado MC, Latorre A, et al Active and secreted IgA-coated bacterial fractions from the human gut reveal an under-represented microbiota core. Sci Rep. 2013;3:3515.

18. Cole JR, Wang Q, Cardenas E, Fish J, Chai B, Farris RJ, et al. The ribosomal database project: improved alignments and new tools for rrna analysis. Nucleic Acids Res. 2009;37:D141-5.

19. Ghannoum MA, Jurevic RJ, Mukherjee PK, Cui F, Sikaroodi M, Naqvi A, et al. Characterization of the oral fungal microbiome (mycobiome) in healthy individuals. PLoS Pathog. 2010;6(1):e1000713.

20. Palm NW, de Zoete MR, Cullen TW, Barry NA, Stefanowski J, Hao L, et al. Immunoglobulin A coating identifies colitogenic bacteria in inflammatory bowel disease. Cell. 2014;158:1000-10. 